\title{
Stable Oscillations in Single Species Growth Models with Hereditary Effects*
}

\author{
DONALD S. COHEN, EVANGELOS COUTSIAS AND JOHN C. NEU \\ Department of Applied Mathematics, California Institute of Technology, Pasadena, California \\ 91125
}

Received 31 May 1978; revised 19 December 1978

\begin{abstract}
We study single species growth models incorporating hereditary effects. Detailed calculations are carried out for a specific model with one delay parameter $T$ as $T$ varies in the entire range $T>0$. Using perturbation and bifurcation techniques, we show that the effect of the hereditary term is that the equilibrium state, which is stable for small values of $T$ (say $T<T_{1}$ ), is unstable for $T_{1}<T<T_{2}$, and regains its stability for large delays $T>T_{2}$. We show that for $T_{1}<T<T_{2}$ a stable oscillatory state, exists which bifurcates from the equilibrium state through an exchange of stability at $T=T_{1}$ and $T=T_{2}$. Numerical computations and graphs of the solutions are given for the solutions in all ranges of $T$.
\end{abstract}

\section{INTRODUCTION.}

Many variations and modifications of Volterra's population equations have been proposed to explain the observed stable steady and oscillatory patterns in population dynamics. These models, incorporating realistic effects, tend to be highly nonlinear, and it is only very recently that sophisticated techniques in bifurcation and perturbation theory and to a lesser extent global analysis have been created which allow a somewhat comprehensive attack on the models. Analytically our approach is a formal multitime scale perturbation method. Not only does the method produce formulas which are immediately interpretable physically, but also the stability of the solutions (and correspondingly the decision of super or subcriticality of bifurcation) is immediately resolved without recourse to further techniques.

* This work was supported in part by the U.S. Army Research Office under contract DAHC-34-68-C-006 and the National Science Foundation under Grant GP-32157X2.

MATHEMATICAL BIOSCIENCES 44:255-268 (1979) 
We study general models incorporating hereditary effects. To further illustrate the method, we carry out calculations for a specific model with one delay parameter $T$. For the specific model chosen we find that the effect of the hereditary (or catabolic) term is that the equilibrium state, which is stable for small values of the delay $T$, (say $T<T_{1}$ ), is unstable for $T_{1}<T<T_{2}$, and regains its stability for large delays $T>T_{2}$. We shall show that for $T_{1}<T<T_{2}$, a stable oscillatory state exists which bifurcates from the equilibrium state through an exchange of stability at $T=T_{1}$ and $T=T_{2}$.

The models and equations to be analyzed are derived in Sec. 2. In Sec. 3 we present our results for a specific model with one delay parameter. Finally, in Sec. 4 we present the multiscale analysis which leads to our results. Among other things, this analysis demonstrates that for our specific model, bifurcation at $T_{1}$ is supercritical and bifurcation at $T_{2}$ is subcritical.

\section{THE MODEL}

We consider a general single species model in which the instantaneous per unit growth rate is given by

$$
\frac{N^{\prime}}{N}=f\left(N, \int_{-\infty}^{t} K(t-s) g(N(s)) d s\right)
$$

Here $N(t)$ is the population at time $t, f$ is an arbitrary function, and the integral term represents a delay (or hereditary) term. The integral term, for example, could model the delayed effect of environmental intoxication due to catabolic effects, or in an entirely different context the replenishment or regeneration time for vegetation for grazing herbivores. The notes [1] by $\mathrm{J}$. M. Cushing consitute an excellent and useful survey of results and problems for integrodifferential equations arising from such effects as heredity.

We shall assume that the kernel $K(\omega)$ is non-negative and smooth for all $\omega \geqslant 0$, and furthermore that $K(\omega)$ decays exponentially as $\omega \rightarrow \infty$. With suitable a priori bounds on the solution of (1) or with suitable conditions on the function $g(N)$, it follows from fundamental polynomial approximation theorems (for example, the completeness of the Laguerre polynomials) that given any $\varepsilon>0$ there exists a function

$$
\tilde{K}(\omega)=\sum_{n=0}^{m} a_{n} \omega^{n} e^{-\omega}
$$

where $m$ may depend upon $\varepsilon$, such that

$$
\left|\int_{-\infty}^{t} K(t-s) g(N(s)) d s-\int_{-\infty}^{t} \tilde{K}(t-s) g(N(s)) d s\right|<\varepsilon
$$


We now replace the original kernel $K(\omega)$ by its approximation $\tilde{K}(\omega)$, so that we can transform (1) into a system of nonlinear differential equations as follows: Let

$$
\begin{aligned}
Z(t) & =\int_{-\infty}^{t} \tilde{K}(t-s) g(N(s)) d s \\
& =\sum_{n=0}^{m} a_{n} \int_{-\infty}^{t}(t-s)^{n} e^{-(t-s)} g(N(s)) d s,
\end{aligned}
$$

and let

$$
Z_{n}(t)=\int_{-\infty}^{t}(t-s)^{n} e^{-(t-s)} g(N(s)) d s .
$$

Then

$$
\frac{d Z_{0}}{d t}=g(N(t))-Z_{0}
$$

and

$$
\frac{d Z_{n}}{d t}=-Z_{n}+n Z_{n-1}, \quad n \geqslant 1 .
$$

Thus,

$$
\frac{d Z}{d t}=a_{0} g(N)-a_{0} Z_{0}+\sum_{n=1}^{m} a_{n}\left(-Z_{n}+n Z_{n-1}\right) .
$$

Therefore, the original equation (1), written as $N^{\prime}=f(N, Z) N$, together with equations (6), (7), and (8), constitutes a set of $m+3$ differential equations in the $m+3$ unknowns $N, Z, Z_{0}, \ldots, Z_{m}$. We assume that the solution of the approximate equation [i.e., the equation (1) with kernel $\tilde{K}$ ] approximates the solution of the original equation. Obvious extensions can clearly handle any number of equations with any number of kernels.

For the study of exchanges of stability via various types of bifurcations the differential system (1), (6), (7), (8) is in some ways more tractable than the original integrodifferential equation. For example, if there is a change of stability via a Hopf bifurcation, then a formal multiscale perturbation method can be employed to analyze the solutions together with resolving stability (i.e., direction of bifurcation). This procedure has been carried out by D. S. Cohen [2] for general systems of equations of the form $Y^{\prime}=$ $F(Y, \lambda)$, where $Y=\left(y_{1}, \ldots, y_{n}\right)$ and $\lambda$ is some parameter. 
For ease of presentation here and in order not to obscure the basic method with lengthy algebraic calculations, we shall now carry out an analysis for the special case

$$
\frac{d N}{d t}=\left[b-a N-d_{0} p_{0}\left(\int_{-\infty}^{t} K(t-s) N(s) d s\right)^{2}\right] N,
$$

where we take the kernel $K(\omega)$ to be

$$
K(\omega)=\frac{\omega}{T^{2}} e^{-\omega / T} .
$$

Here $a, b, d_{0}$, and $p_{0}$ are prescribed positive constants. Equations (9) and (10) constitute Cushing's modification [1] of a model of A. Borsellino and V. Torre [3]. Borsellino and Torre modeled and numerically studied a situation in which delayed growth rate responses are related to accumulating environmental intoxicants due to catabolic waste residuals where the pollutants in turn are related to past population sizes. The modification (9), (10) constitutes a convenient mathematical simplification which retains all qualitative features but which is analytically simpler to manipulate. Derived directly but in a more artificial situation, consider

$$
\frac{d N}{d t}=\left[b-a N-d \int_{-\infty}^{t} K(t-s) N(s) d s\right] N,
$$

which in the absence of the integral term is the well-known logistic equation. Suppose that the coefficient $d$, which measures the magnitude of the response of the growth rate to past population sizes, is proportional to past population sizes; that is, assume that

$$
d=p_{0} \int_{-\infty}^{t} K(t-s) N(s) d s
$$

When (12) is substituted into (11), we obtain (9). The kernel (10) is positive and attains its maximum at $\omega=T$. Thus, its major influence on the growth rate response at any time $t$ is due to population density at the previous time $t-T$. For this reason we call $T$ the delay.

By using the reduction introduced in the beginning of this section we find that the problem (9), (10) is equivalent to the system

$$
\begin{aligned}
N^{\prime} & =\left(b-a N-d_{0} p_{0} z^{2}\right) N, \\
z^{\prime} & =\frac{-1}{T} z+\frac{1}{T^{2}} y, \\
y^{\prime} & =N-\frac{1}{T} y,
\end{aligned}
$$


where

$$
z(t)=\int_{-\infty}^{t} K(t-s) N(s) d s
$$

In Sec. 4 we shall apply our bifurcation and perturbation theory to the system (13). First, however, we present the results of this analysis in Sec. 3.

\section{RESULTS}

Note that the kernel $K(\omega)$ has been normalized so that its integral is one. Then, it is easy to show that the model (9), (10) has a unique equilibrium $e>0$ given by the positive root of the quadratic equation $d_{0} p_{0} e^{2}+a e-b=$ 0 ; that is,

$$
e=\frac{-a+\left(a^{2}+4 d_{0} p_{0} b\right)^{1 / 2}}{2 d_{0} p_{0}}>0 .
$$

J. M. Cushing [1] has analyzed the (linearized) stability of $e$. We briefly present his results, since we need them to derive our own. By linearizing (9) about $e$ in the usual way, Cushing shows that $e$ is stable if and only if $H \equiv a^{2} e^{2} T+\left(2 a e-d_{0} p_{0} e^{2}\right) T+1>0$, and unstable if and only if $H<0$. Now, $H>0$ for all $T$ if $d_{0} p_{0}<4 a$, but if $d_{0} p_{0}>4 a$, then $H<0$ if and only if $T_{1}<T<T_{2}$, where $T_{i}(i=1,2)$ are the two positive real roots of the quadratic equation $H=0$. Therefore, if the pollution coefficient $p_{0}$ is large, the equilibrium $e$ is stable for small delays $T<T_{1}$, unstable for $T_{1}<T<T_{2}$, and stable again for large delays $T>T_{2}$. Furthermore, $e$ decreases as $p_{0}$ increases, and $T_{1}$ is a monotonically decreasing (to zero) function of $p_{0}$, so that the equilibrium state $e$ loses its stability for shorter delays as $p_{0}$ increases.

We show in Sec. 4 that $T=T_{1}$ and $T=T_{2}$ are bifurcation points at which there is an exchange of stability between the equilibrium value $e$ and an oscillatory state. More precisely, as $T$ is increased from zero, the equilibrium state $N(t) \equiv e$ is stable until $T=T_{1}$. At $T_{1}$ the equilibrium $e$ loses its stability, and a stable small amplitude oscillation (about $e$ ) is set up. As $T$ is increased further, the oscillation amplitude grows for a while and then decreases again until the oscillation disappears at $T=T_{2}$, at which value $e$ becomes the unique stable state again. Thus, in $T_{1}<T<T_{2}$ the population $N(t)$ evolves to a stable oscillation, as illustrated in the bifurcation diagram sketches in Fig. 1.

The analysis to support our general results is given in Sec. 4. For the specific parameter values $b=5, a=1, d_{0} p_{0}=5$, we have computed some 


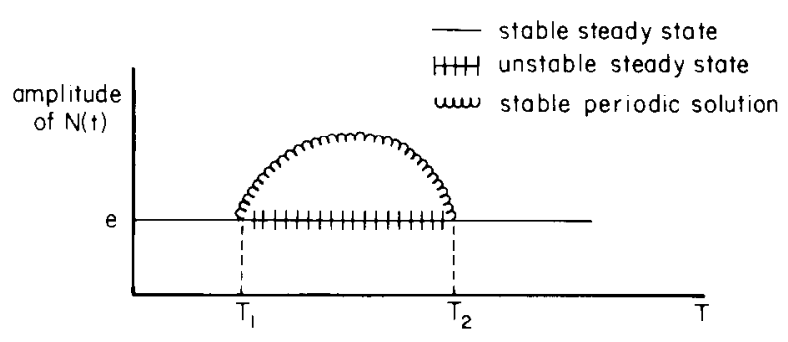

FIG. 1.

typical profiles for $N(t)$ from the system of equations (13). Each run has the initial conditions $N(0)=y(0)=z(0)=0.01$, which is very close to the unstable steady state at $(N, y, z)=(0,0,0)$. Given the value of $T$, which measures the delay lime of the kernel $K(\omega)$, we observe the subsequent evolution of $N(t)$ to the steady state with $N=e$ or a limit cycle. For $b=5, a=1, d_{0} p_{0}=5$, the steady state with $N=e$ is stable if $T<T_{1}=0.54350$ or $T>T_{2}=2.24653$, and unstable if $T_{1}=0.54350<T<2.24653=T_{2}$. Figures 2 and 3 show $N(t)$ for $T=0.5$ and $T=2.5$. In both cases, we observe decay to the steady state $N=e=0.90499$. Figures 4-9 show $N(t)$ for $T$ with various values between $T_{1}$ and $T_{2}$. In each case, $N(t)$ evolves to a periodic solution. Table 1 records the observed amplitudes of the oscillations vs. $T$. It is of interest to note that these results are consistent with the bifurcation diagram in Fig. 1.

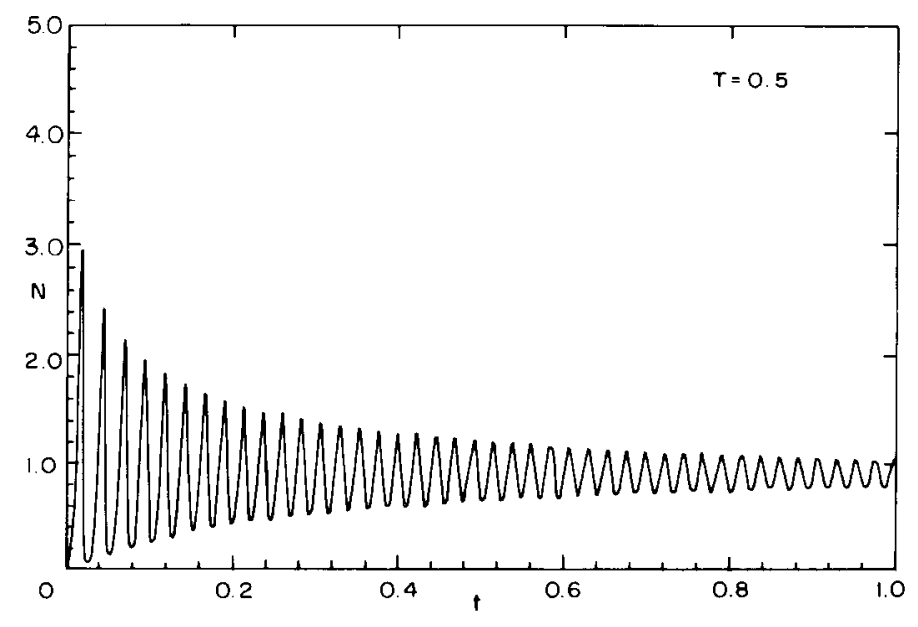

Fig. 2. 


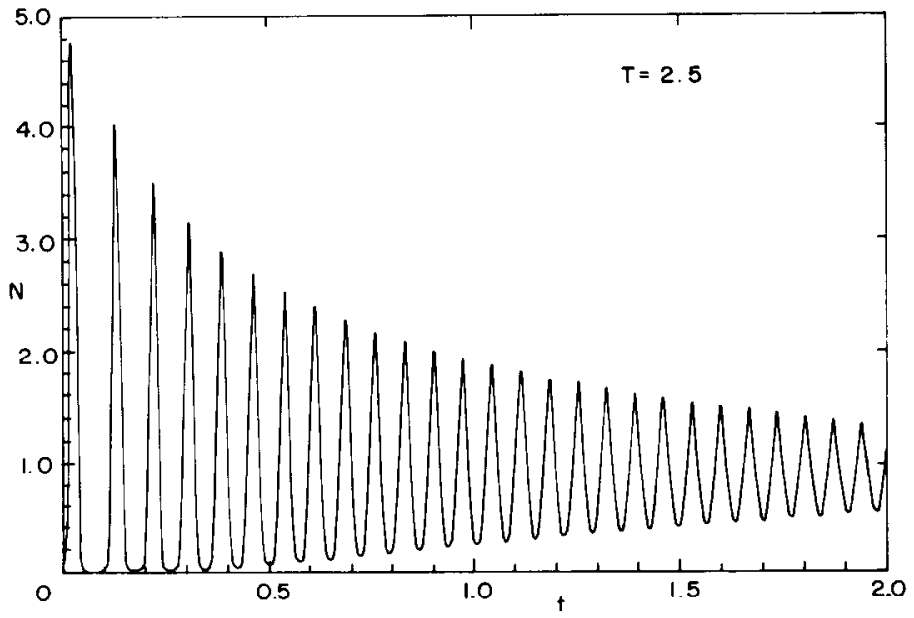

FIG. 3.

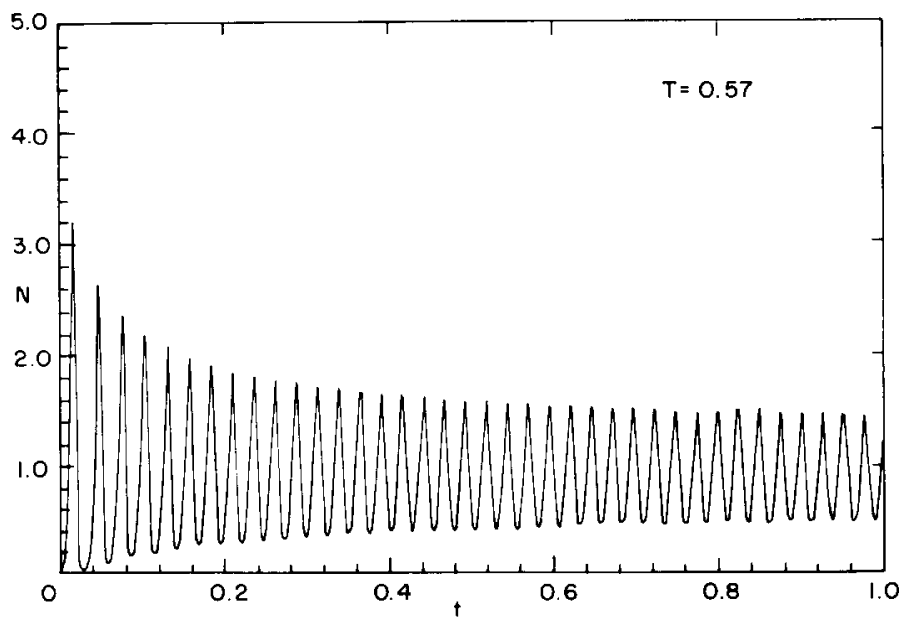

FIG. 4. 


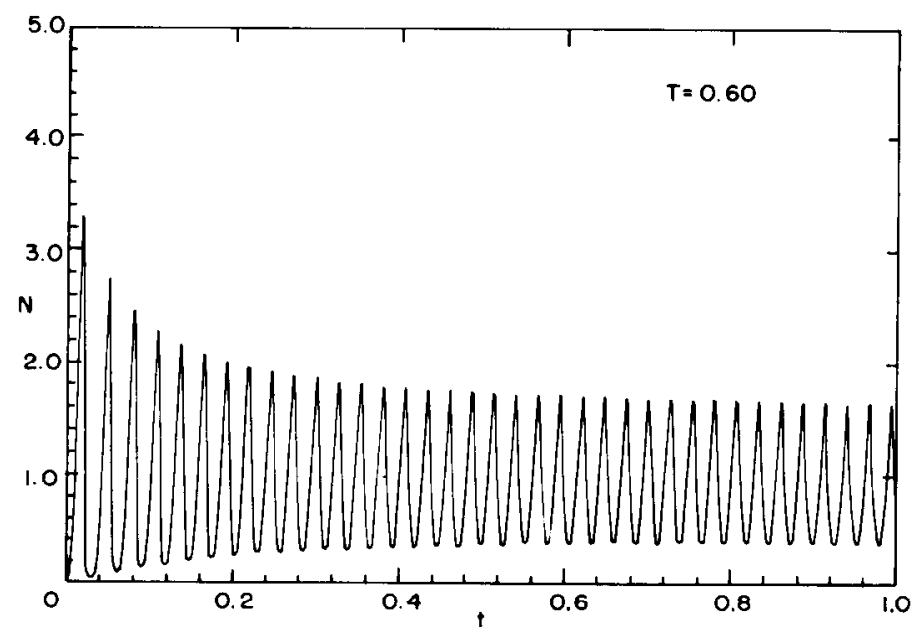

FIG. 5.

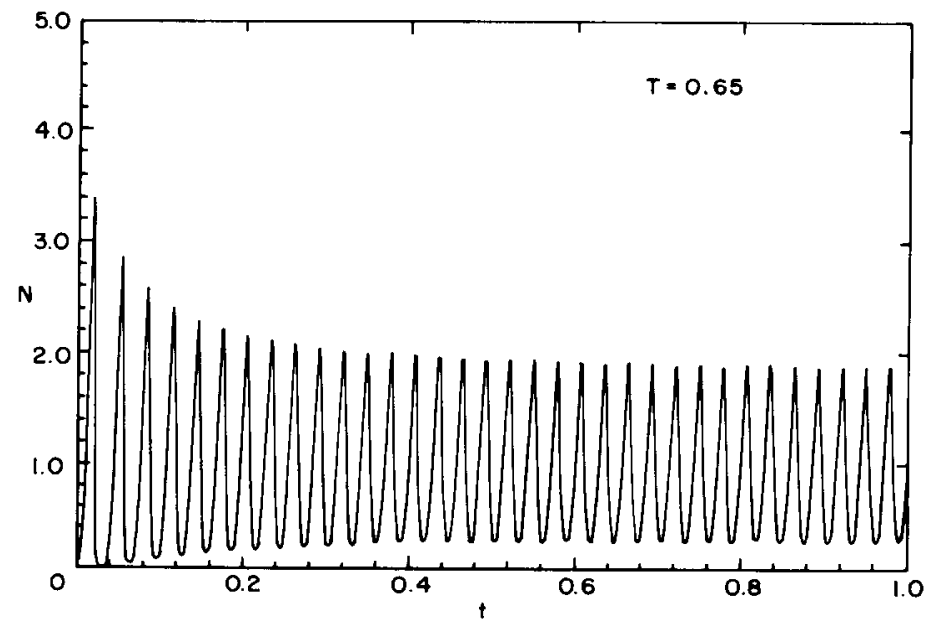

Fig. 6. 


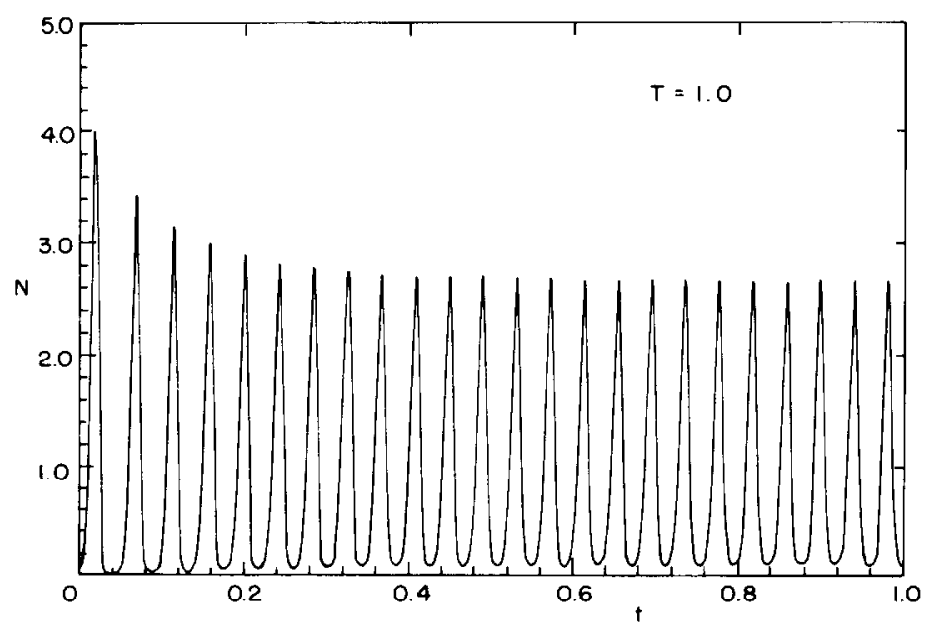

FIG. 7.

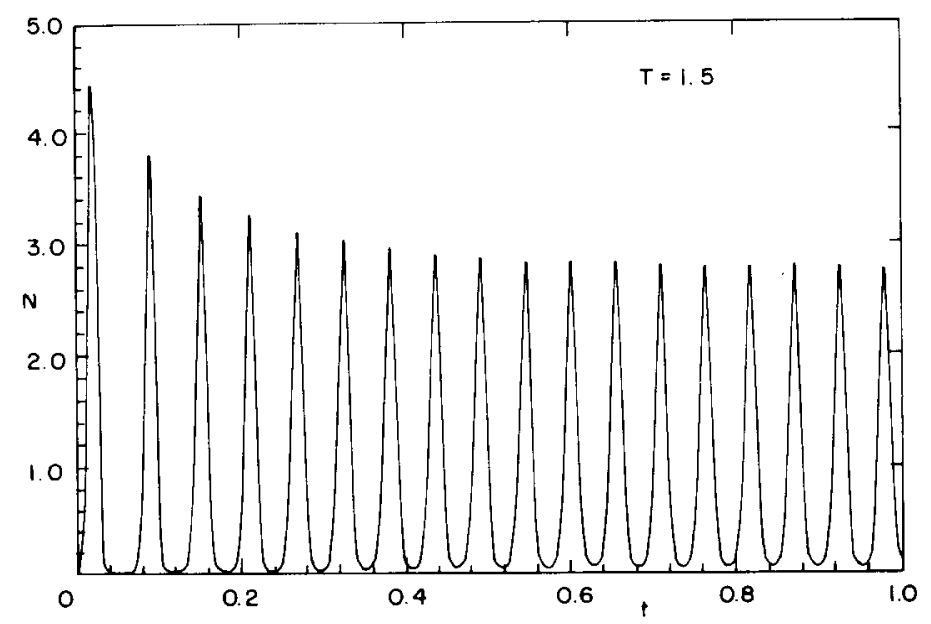

FIG. 8. 


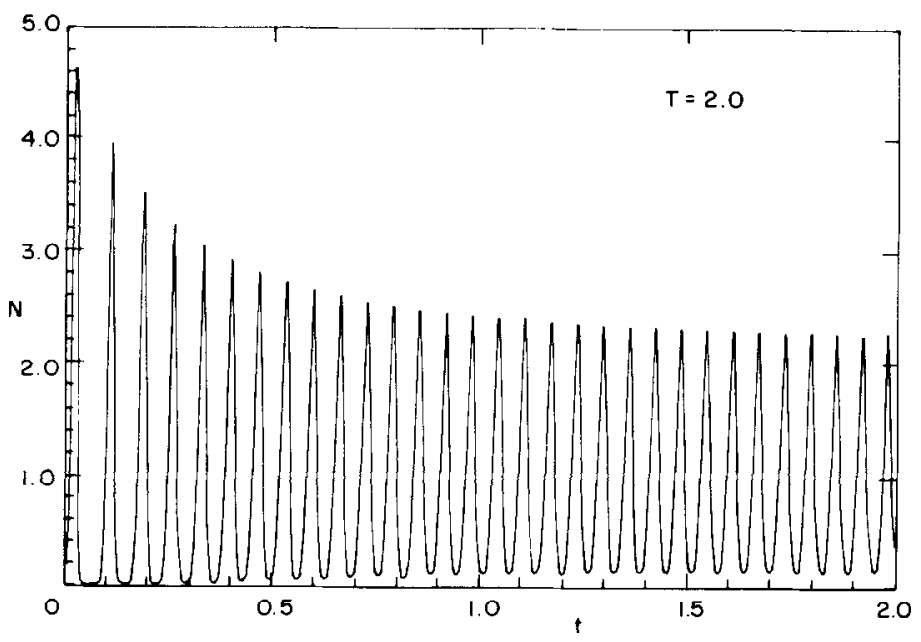

FIG. 9.

TABLE 1

\begin{tabular}{lc}
\hline \multicolumn{1}{c}{$T$} & Amplitude \\
\hline 0.54350 & 0 \\
0.57 & 1.0 \\
0.60 & 1.3 \\
0.65 & 1.6 \\
1.0 & 2.6 \\
1.5 & 2.8 \\
2.0 & 2.3 \\
2.24653 & 0 \\
\hline
\end{tabular}

\section{THE BIFURCATION THEORY}

Write the system (13) as

$$
\frac{d Y}{d t}=F(Y, T)
$$

where

$$
Y \equiv\left(\begin{array}{c}
N \\
z \\
y
\end{array}\right), \quad F(Y, T) \equiv\left(\begin{array}{l}
\left(b-a N-d_{0} p_{0} z^{2}\right) N \\
\frac{-1}{T} z+\frac{1}{T^{2}} y \\
N-\frac{1}{T} y
\end{array}\right)
$$


The system (15) has an equilibrium value at the point $Y_{0}(T) \equiv(N, z, y)=$ (e,e,Te); that is, $F\left(Y_{0}(T), T\right)=0$. To determine the (linearized) stability of the steady state $Y_{0}(T)$, we linearize (15) around this steady state. In the standard manner we obtain a system of linear ordinary differential equations with constant coefficients, the solutions of which are linear combinations of exponentials of the form $e^{\mu_{i} t}, i=1,2,3$, where the $\mu_{i}$ are the eigenvalues of the matrix $F_{Y}\left(Y_{0}(T), T\right)$. It is easy to show that there is one real negative eigenvalue and a complex conjugate pair $\alpha(T) \pm i \beta(T)$, where $\alpha(T)<0$ for $T<T_{1}$ and for $T>T_{2}, \alpha\left(T_{1}\right)=0=\alpha\left(T_{2}\right)$, and $\alpha(T)>0$ for $T_{1}<T<T_{2}$. Thus, the system (15) undergoes a simple Hopf bifurcation at $T=T_{1}$ and at $T=T_{2}$.

We now cmploy a multiscalc perturbation procedure to carry out a nonlinear stability analysis to support the claims of Sec. 3. Our analysis follows that of D. S. Cohen and J. P. Keener [2, 4]. Since all the detailed manipulations are carried out in [2] and [4], we shall simply set up and outline the approach for the present problem and refer the reader to those papers for the details.

Let $Z(t, T)=Y(t, T)-Y_{0}(T)$ and expand the right hand side of (15) around the equilibrium solution $Y_{0}(T)$ to write (15) as

$$
\frac{d Z}{d t}=F_{Y}\left(Y_{0}(T), T\right) Z+H(Z),
$$

where $H(Z)$ denotes the remainder after the linear terms and hence contains no linear terms near $Y_{0}(T)$. Now, let $Z=B X$, where the (constant) matrix $B$ is yet to be specified. Then (17) becomes

$$
\frac{d X}{d t}=B^{-1} F_{Y}\left(Y_{0}(T), T\right) B X+B^{-1} H(B X) .
$$

At $T=T_{1}$ (a similar analysis holds at $T=T_{2}$ ) we can choose $B$ such that

$$
B^{-1} F_{Y}\left(Y_{0}(T), T\right) B=\left(\begin{array}{ccc}
0 & \beta\left(T_{1}\right) & 0 \\
-\beta\left(T_{1}\right) & 0 & 0 \\
0 & 0 & \mu_{3}
\end{array}\right) \equiv P,
$$

where $\mu_{3}$ denotes the real negative eigenvalue at $T=T_{1}$. Define a small parameter $\varepsilon^{2}=T-T_{1}$ so that we are slightly into the region where the steady state $Y_{0}(T)$ is unstable. We shall show that there exist stable oscillatory solutions of amplitude $O(\varepsilon)$ for $T>T_{1}$. (At $T=T_{2}$ we would define our small parameter as $\varepsilon^{2}=T_{2}-T$, so that the multiscale procedure would establish evolution onto a bifurcating subcritical branch for the present problem.) Now since

$$
F_{Y}\left(Y_{0}(T), T\right)=F_{Y}\left(Y_{0}\left(T_{1}\right), T_{1}\right)+O\left(\left|T-T_{1}\right|\right),
$$


we can choose $B$ such that

$$
B^{-1} F_{Y}\left(Y_{0}(T), T\right) B=P+\varepsilon^{2} A,
$$

where the matrix $A$ can be singular or even identically zero. Therefore, we choose $B$ such that (19) is satisfied, when $P$ is given in (19). Then the system (15) becomes

$$
\frac{d X}{d t}=P X+\varepsilon^{2} A X+G(X),
$$

where $G(X)=B^{-1} H(B X)$ and where

$$
G(X)=\left(\begin{array}{l}
g_{1}(X) \\
g_{2}(X) \\
g_{3}(X)
\end{array}\right), \quad g_{i}(0)=\left(\frac{\partial g_{i}}{\partial x_{j}}\right)(0)=0, \quad i, j=1,2,3 .
$$

By using multiscale perturbation methods, Cohen and Keener $[4,5]$ have shown that to leading order in $\varepsilon$ the solution $X(t)=\left(x_{1}(t), x_{2}(t), x_{3}(t)\right)$ of $(21)$ possesses the expansion

$$
\begin{aligned}
& x_{1}(t) \sim \varepsilon R(\tau) \sin \left[\beta\left(T_{1}\right) t^{*}+\phi(\tau)\right], \\
& x_{2}(t) \sim \varepsilon T(\tau) \cos \left[\beta\left(T_{1}\right) t^{*}+\phi(\tau)\right], \\
& x_{3}(t) \sim \varepsilon D(\tau) e^{\mu_{3} t^{*}},
\end{aligned}
$$

where $R$ and $\phi$ are the slowly varying amplitude and phase on the "slow time" scale $\tau=\varepsilon^{2} t$, and $t^{*}=\left[1+O\left(\varepsilon^{2}\right)\right] t$ represents the "fast time." Furthermore, it is shown in [4], [5] that $K$ satisfies the equation

$$
\frac{d R}{d \tau}=\alpha R-\gamma R^{3},
$$

the solution of which is

$$
R^{2}(\tau)=\frac{\alpha}{\gamma} \frac{1}{1+c e^{-\alpha \tau}},(c=\text { constant }) .
$$

Here $\alpha$ and $\gamma$ are constants depending on parameters and derivatives of nonlinearities in the original problem. The formulas for $\alpha$ and $\gamma$ are very lengthy, but they have been worked out and are easy to use. They are given in [4], and we find for the present example that $\alpha>0$ and $\gamma>0$.

Equations (23) and (25) show that $X(t)$ approaches a limit cycle in the $\left(x_{1}, x_{2}\right)$-plane of amplitude $(\alpha / \gamma)^{1 / 2} \varepsilon$. Upon going back through our transformations from (21) to our original problem (15), we obtain the bifurcation result stated in Sec. 3. The analysis of this section establishes the bifurcation 
results at $T=T_{1}$ and at $T=T_{2}$. Note that the multiscale procedure establishes the direction of bifurcation, since on either side of the bifurcation it shows the evolution of an arbitrary (small) perturbation. In the present problem the scaling $\varepsilon^{2}=T-T_{1}>0$ establishes supercritical bifurcation at $T_{1}$. In other problems it could be necessary to scale with $\varepsilon^{2}=T_{1}-T>0$ to establish subcritical bifurcation. This is determined by successful derivations of meaningful slow time modulation amplitudes $R(\tau)$. The global behavior for the present example is deduced by combining the numerical computations with the perturbation expansion; this rules out possible turning of the branches far away from the stability boundaries.

\section{REFERENCES}

1 J. M. Cushing, Integrodifferential Equations and Delay Models in Population Dynamics, Springer-Verlag Lecture Note Series on Biomathematics, 1977.

2 D. S. Cohen, Bifurcation from multiple complex eigenvalues, J. Math. Anal. Appl. 57:505-521 (1977).

3 A. Borsellino and V. Torre, Limits to growth from Volterra Theory of population, Kybernetik 16:113-118 (1974).

4 D. S. Cohen and J. P. Keener, Multiplicity and stability of oscillatory states in a continuous stirred tank reactor with exothermic-consecutive reaction $A \rightarrow B \rightarrow C$, Chem. Eng. Sci. 31:115-122 (1976).

5 D. S. Cohen and J. P. Keener, Oscillatory processes in the theory of particulate formation in super-saturated chemical solutions, SIAM J. Appl. Math. 28:307-318 (1975). 